parameters $\alpha$ from the minimum principle

$$
\operatorname{Iin}_{\alpha_{1}, \cdots \alpha_{N}} \sum_{A=1}^{M}\left[\int \sigma_{i j}^{A} \epsilon_{i j} d V-\int u_{i} \sigma_{i j}^{A} n_{j} d S\right]^{2} .
$$

Alternatively, the parameters $\alpha$ could be determined from a minimax principle, such as

$$
\operatorname{Min}_{\alpha_{1}, \cdots \alpha_{N}}\left\{\operatorname{Max}_{\beta_{1}, \cdots \beta_{M}}\left[\int\left(\sum_{A=1}^{M} \beta_{A} \sigma_{i i}^{A}\right) \epsilon_{i j} d V-\int u_{i}\left(\sum_{A=1}^{M} \beta_{A} \sigma_{i i}^{A}\right) n_{i} d S\right]^{2}\right\},
$$

where the parameters $\beta$ must satisfy $\sum_{A=1}^{M} \beta_{A}^{2}=1$.

\title{
A NOTE ON LAMINAR AXIALLY SYMMETRIC JETS*
}

BY MARK BERAN (Wellesley, Mass.)

Summary. It is shown that there is no stream function of the form $\psi=r f(\theta)$, that is compatible with the complete Navier-Stokes equations, which represents a jet issuing from a small circular hole in an axially symmetric cone.

The asymptotic velocity field of a laminar viscous jet is generally accepted to have a stream function of the form $\psi=r f(\theta)$, corresponding to self-similar flow (Schlichting [1], Squire [2], and Yatseev [3]). The authors referred to have based their discussion on the fact that this assumption of self-similarity is compatible with both the boundary layer equations, and with the full Navier-Stokes equations.

The purpose of this note is to establish a serious shortcoming of such models. It is shown that there is no continuously differentiable velocity field associated with a stream function of the form $\psi=r f(\theta)$, which satisfies the Navier-Stokes equations and also adheres to a conical wall $\theta=\alpha>0$.

Specifically, if $\psi=r f(\theta)$, then the velocity components in the $r$ and $\theta$ directions are respectively [4]

$$
\begin{gathered}
u_{r}=\left[\frac{1}{r \sin \theta}\right] \frac{d f}{d \theta}, \\
u_{\theta}=\left[\frac{-1}{r \sin \theta}\right] f .
\end{gathered}
$$

The Navier-Stokes equations are equivalent to [5]

$$
f^{2}=4 \nu \cos \theta f-2 \nu \sin \theta \frac{d f}{d \theta}-2\left(c_{1} \cos ^{2} \theta+c_{2} \cos \theta+c_{3}\right)
$$

for suitable constants $c_{1}, c_{2}, c_{3}$. We shall show that there is no solution of (3) which (i) makes $u_{r}$ and $u_{\theta}$ continuous for $r>0$, and (ii) satisfies $u_{r}(\alpha)=u_{\theta}(\alpha)=0$, for 0 $<\alpha \leq \pi$.

To show this, we also consider the differentiated form of (3), which is

$$
\frac{-f}{\sin \theta} \frac{d f}{d \theta}=2 f-2 \sin \theta \frac{d}{d \theta}\left[\frac{1}{\sin \theta} \frac{d f}{d \theta}\right]-\left(2 c_{1} \cos \theta+c_{2}\right) \text {. }
$$

*Received June 28, 1955; revised manuscript received October 5, 1955. The work on this paper was partly supported by Contract N5ori-07634 with the Office of Naval Research. 
The boundary conditions implied by conditions (i) and (ii) are:

For Eq. (4): (a) $u_{\theta}=0, u_{r}$ finite, when $\theta=0$.

(b) $u_{r}=u_{\theta}=0$, when $\theta=\alpha$.

For Eq. (5): (c) $u_{\theta}=0, u_{r}$ finite, $\partial u_{r} / \partial \theta$ finite, when $\theta=0 .^{*}$

These yield respectively the equations:

$$
\begin{gathered}
c_{1}+c_{2}+c_{3}=0, \\
c_{1} \cos ^{2} \alpha+c_{2} \cos \alpha+c_{3}=0, \\
2 c_{1}+c_{2}=0 .
\end{gathered}
$$

These have $c_{1}=c_{2}=c_{3}=0$ as their only solution.

As Squire [5] has shown, the general solution of Eq. (4) with $c_{1}=c_{2}=c_{3}=0$ is:

$$
f=\frac{2 \nu \sin ^{2} \theta}{a+1-\cos \theta}
$$

where $a$ is an arbitrary constant.

Referring again to the boundary condition $u_{r}(\alpha)=u_{\theta}(\alpha)=0$, we see that there is no finite value of $a(a=\infty$ yields a satisfactory but trivial solution) which satisfies this boundary condition, no matter what value of $\alpha$ is chosen.

Thus we have shown that there is no non-trivial solution of the form $\psi=r f(\theta)$ that is compatible with the Navier-Stokes equations and the boundary conditions (i) and (ii).

The author wishes to thank Prof. Garrett Birkhoff for suggesting the problem and for his helpful advice.

\title{
REFERENCES
}

[1] S. Goldstein (ed.), Modern developments in fluid dynamics, vol. 1, Oxford, 1938, p. 147. Also H. Schlichting, Grenzschichtstheorie, Karlsruhe

[2] H. B. Squire, Phil. Mag. 43, 942-5 (1952)

[3] V. L. Yatseev, Zh. eksp. teor. fiz. 20, 1031-34 (1950)

[4] S. Goldstein (ed.), Modern developments in fluid dynamics, vol. 1, Oxford, 1938, pp. 104 and 115

[5] H. B. Squire, Quart. J. Mech. Appl. Math. 4, 321-29 (1950)

*It is assumed that when these boundary conditions are substituted in Eqs. (4) and (5) that the limit $\theta \rightarrow 0$ is taken, since $\theta=0$ is a singular point in the spherical polar coordinate system.

\section{HEAT CONDUCTION IN SEMI-INFINITE SOLID IN CONTACT WITH LINEARLY INCREASING MASS OF FLUID*}

\author{
By C. C. CHAO AND J. H. WEINER (Columbia University)
}

Introduction. Problems of transient heat conduction in which the surface of a solid is in contact with a well-stirred fluid have been the subject of numerous investigations. ${ }^{1}$ In all previous cases studied, the mass of the fluid has been considered constant. However, it is sometimes of interest to know the temperature in the solid and fluid

*Received August 9, 1955.

${ }^{1} \mathrm{~A}$ review of previous work is found in [-1], pp. 16-17. 